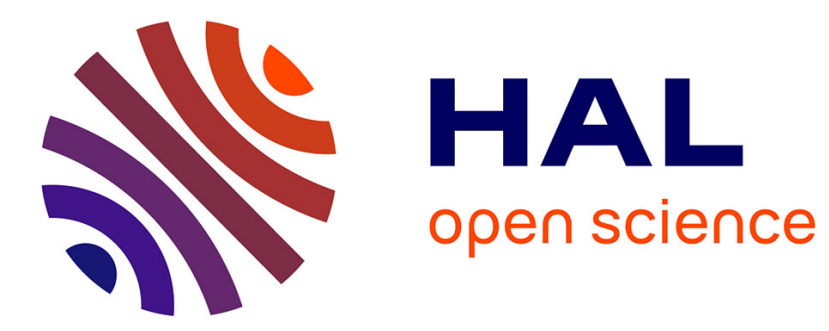

\title{
Vitesse, déplacement et transparence des êtres fantomatiques et des personnes autistes
}

Chantal Lheureux-Davidse

\section{To cite this version:}

Chantal Lheureux-Davidse. Vitesse, déplacement et transparence des êtres fantomatiques et des personnes autistes. Cliniques méditerranéennes, 2012, L'enfant et ses fantômes, 2 (86), pp.71 - 83. 10.3917/cm.086.0071 . hal-01513814

\section{HAL Id: hal-01513814 https://hal.science/hal-01513814}

Submitted on 25 Apr 2017

HAL is a multi-disciplinary open access archive for the deposit and dissemination of scientific research documents, whether they are published or not. The documents may come from teaching and research institutions in France or abroad, or from public or private research centers.
L'archive ouverte pluridisciplinaire HAL, est destinée au dépôt et à la diffusion de documents scientifiques de niveau recherche, publiés ou non, émanant des établissements d'enseignement et de recherche français ou étrangers, des laboratoires publics ou privés. 


\section{VITESSE, DÉPLACEMENT ET TRANSPARENCE DES ÊTRES FANTOMATIQUES ET DES PERSONNES AUTISTES}

Chantal Lheureux-Davidse

\section{ERES | «Cliniques méditerranéennes »}

2012/2 n 86 | pages 71 à 83

ISSN 0762-7491

ISBN 9782749234359

Article disponible en ligne à l'adresse :

http://www.cairn.info/revue-cliniques-mediterraneennes-2012-2-page-71.htm

\section{Pour citer cet article :}

Chantal Lheureux-Davidse, "Vitesse, déplacement et transparence des êtres fantomatiques et des personnes autistes », Cliniques méditerranéennes 2012/2 ( ${ }^{\circ}$ 86), p. 71-83.

DOI $10.3917 / \mathrm{cm} .086 .0071$

Distribution électronique Cairn.info pour ERES.

(C) ERES. Tous droits réservés pour tous pays.

La reproduction ou représentation de cet article, notamment par photocopie, n'est autorisée que dans les limites des conditions générales d'utilisation du site ou, le cas échéant, des conditions générales de la licence souscrite par votre établissement. Toute autre reproduction ou représentation, en tout ou partie, sous quelque forme et de quelque manière que ce soit, est interdite sauf accord préalable et écrit de l'éditeur, en dehors des cas prévus par la législation en vigueur en France. Il est précisé que son stockage dans une base de données est également interdit. 


\section{Chantal Lheureux-Davidse}

\section{Vitesse, déplacement et transparence des êtres fantomatiques et des personnes autistes}

Les fantômes tels que nous nous les figurons sont dotés de facultés particulières. Leur transparence et leur inconsistance charnelle leur permettent de se déplacer dans une direction sans que rien ne les entame ou ne modifie leur trajet car ils traversent les murs, les objets ou les personnes dans l'espace sans tenir compte des limites matérielles. Ils prennent une allure humaine mais incolore comme un mort ou peuvent être figurés par une forme inquiétante en mouvement comme un drap blanc qui entourerait un espace vide laissé par un corps absent, avec des trous sans fond à la place des yeux.

Leur volume apparent peut se réduire en un instant à une forme plate en deux dimensions avant de retrouver l'apparence externe d'un volume. Ils ne font plus partie des vivants tout en en ayant l'allure, mais sans chair. Ils tiennent par eux-mêmes sans support, comme en suspension, ils ne touchent pas le sol sans pour autant être suspendus ni appuyés puisqu'ils n'ont souvent pas de forme dans le bas de leur allure corporelle.

Ils vont et viennent dans des mouvements fluides comme au ralenti. Leurs déplacements ne sont pas accordés à ceux des communs des mortels, qui eux se déplacent au rythme de la marche. Rien ne semble pouvoir les arrêter. Personne ne pourrait modifier leurs trajets.

Certains habitent les lieux des vivants telles des âmes sans chair. D'autres, par leurs déplacements et leurs mouvements, animent les lieux où les morts reposent dans l'immobilité.

Ils peuvent surgir, disparaître ou se déplacer spontanément sans que cela soit prévisible, ce qui les rend très inquiétants. Leur présence n'est

Chantal Lheureux-Davidse, maître de conférences en psychopathologie à l'université Paris 7, 59 rue du Temple, F-75004 Paris - chantal.lheureuxdavidse@laposte.net 
jamais certaine. Leur autonomie de mouvement dans leurs déplacements avec leur allure mêlant la forme humaine et l'informe peut nous hanter ou même nous terroriser. C'est cette paradoxalité entre le vivant et le mort ou entre l'immobilité et l'animé qui est perturbante.

Notre hantise pourrait être qu'ils entrent en collision avec nous, les vivants, de façon imprévisible, en nous effleurant ou en traversant notre corps. Mais l'angoisse si terrifiante de les côtoyer ne deviendrait-elle pas réanimatrice d'éprouvés corporels?

Ils inquiètent les humains qui n'ont pu supporter ni pu intérioriser la disparition d'un être cher. L'identification adhésive à des figures parentales de défunt qui sont aussi inquiétantes potentiellement qu'une identification aux fantômes occupe toute parcelle de l'espace tout en abolissant la séparation, c'est-à-dire la distance nécessaire pour se sentir en sécurité dans son corps.

\section{LA COMPAGNIE DES FANTÔMES}

Certains enfants sont les dépositaires de ces fantômes de la famille sans être au courant de cet héritage, tout en y participant. Paul-Claude Racamier (1992) nous a souvent parlé des deuils exportés et des deuils gelés. Des familles semblent ainsi davantage habitées par les morts jamais tout à fait morts que par les vivants qui ne remplaceront jamais les personnes disparues. Car vivre avec les morts ou comme un mort vivant sauve parfois d'un effondrement catastrophique qui entraînerait des angoisses d'anéantissement. La compagnie des fantômes semble être alors autant nécessaire que terrifiante. Il arrive qu'un enfant naisse dans ce contexte d'un deuil trop douloureux pour la famille, que l'un des parents ne peut entamer et qui imprègne l'atmosphère des relations. La douleur de la séparation est si intense que le côté vivant du bébé ne suffit pas à prendre le devant de la scène dans l'économie psychique parentale. Quand la personne défunte réapparaît comme une personne toujours vivante et animée, de façon hallucinatoire, dans les fantasmes ou dans les rêves, ou si elle est occultée pour ne pas entamer un véritable travail de deuil, la question du mouvement ne fait que pointer l'absence irréversible et insoutenable du contact vivant de son corps et l'absence d'un échange de regard vivant, mobile et contenant.

Le regard absent est comme un regard aspiré dans un trou noir sans fond, selon l'expression de Frances Tustin (1986). Le bébé dans sa spontanéité vivante ne peut pas toujours trouver un accordage joyeux avec un parent endeuillé. Il essaie malgré tout de le rejoindre, au prix de passer dans un état provisoire d'inexistant. Il évite le regard en direct et il se met dans un état flottant qui fait résonance avec celui du parent endeuillé ou déprimé, faute de pouvoir se connecter à une relation vivante et spontanée. 
L'enfant apprend à faire le mort comme s'il habitait le pays des fantômes, et parfois dans le seul accordage qu'est le silence. Se sentir relié dans l'inexistence avec un parent endeuillé ou avec l'environnement non humain vient colmater la perte d'un miroir identifiant parental vivant.

Si l'enfant a une potentialité autistique, ses troubles sensoriels l'entraînent à se démanteler pour tenter de s'apaiser par le processus de clivage. Sa dispersion psychique le déconnecte de tout accordage possible. L'enfant autiste rejoint l'environnement non humain dans un état flottant. Cette désynchronisation a un effet déprimant pour l'enfant comme pour son entourage.

L'histoire de Léa en est une illustration. Je mettrai en évidence la question des déplacements dans l'espace en m'inspirant de nos figurations des fantômes.

\section{DEUIL, DOULEUR ET DÉNI DES ORIGINES}

Léa est le deuxième enfant d'un couple vivant en France originaire d'un pays lointain. Toute la famille de madame est restée dans son pays. Avant même la conception de Léa, madame perd sa propre mère juste avant l'été, quand son premier enfant a 2 ans. Elle apprend ensuite que son père doit être amputé d'une jambe suite à une longue maladie. Madame va passer tout l'été auprès de lui en laissant en France son mari et son premier enfant. Pendant ce temps, monsieur s'est senti abandonné et comme en rivalité avec le père de sa femme.

À son retour en France, elle est encore très choquée d'avoir simultanément perdu sa mère et vu puis laissé son père dans un état si grave. Ses pensées sont davantage tournées vers ses propres parents que vers son mari et son enfant.

Un mois plus tard, madame ne se sent pas très bien. Son médecin lui demande si elle n'est pas enceinte. Un test révèle une grossesse d'un mois. Elle a très vite été enceinte dès son retour en France. Monsieur imagine que le bébé n'est pas de lui mais sans doute d'un amant avec qui sa femme aurait convolé pendant l'été lorsqu'elle était auprès de son père malade. Pendant toute sa grossesse, madame est dans la douleur du déni de paternité de son mari pour ce bébé qu'elle attend ; elle est dans un état de sidération, de chaos et de dépression. Un mois avant terme, madame met au monde Léa. Son mari imagine que le bébé n'est pas du tout prématuré, confirmant ses fantasmes que le bébé ne serait pas de lui. Il quitte définitivement femme et enfants. Madame est en dépression profonde.

Quand monsieur apprend que Léa est diagnostiquée autiste, cela verrouille son déni de paternité, car, dit-il, il n'y a pas ce genre de maladie 
dans sa branche familiale. Monsieur ne pourra jamais se préoccuper de Léa lors des week-ends qui lui seront attribués par la suite pour recevoir ses deux enfants. C'est le fils aîné qui protégera sa petite sœur du rejet paternel pendant des années.

Madame s'occupera au mieux de ses deux enfants et les élèvera seule pendant vingt ans. Elle essaiera malgré cette situation douloureuse de maintenir le lien entre les enfants et leur père sans le disqualifier.

\section{ABSENCE D'ACCORDAGE OU DE MANIFESTATION ET TRANSPARENCE}

À son arrivée en IME, Léa a 8 ans. Je la vois comme une toute petite fille qui en paraît à peine 4 , sans aucune réaction à toute stimulation ou à notre présence. Je l'ai suivie pendant douze ans à raison d'une séance par semaine. Elle n'a aucun langage verbal, aucun son ne sort de sa gorge. Au mieux, elle forme des bulles de salive entre ses lèvres, le regard dans le vide. Pour déglutir, elle avance sa tête péniblement. La plupart du temps sa salive s'écoule en dehors de sa bouche sans qu'elle semble s'en apercevoir.

Elle ne s'ajuste pas à l'autre dans le regard, ni dans sa tonicité posturale. Son visage est inhabité, comme gelé, sans aucune expression. Aucune émotion ne transparaît. Elle ne réagit pas si on l'interpelle. Des examens médicaux n'ont pas décelé de cécité physiologique, ni de surdité. Elle présente des stéréotypies avec ses doigts devant les yeux et passe des heures à se balancer d'avant en arrière.

Elle reste posée comme un " paquet » et ne prend aucune initiative ni pour se lever ni pour s'asseoir. Elle ne sait pas se déplacer seule. Dans la journée elle s'automaintient parfois dans une carapace musculaire interne qui rend sa démarche raide. Cela lui bloque sa digestion et diminue sa capacité respiratoire.

Notre sentiment d'inutilité pour modifier son état est souvent désespérant. Son fonctionnement paraît purement autistique car elle ne réagit apparemment à rien de ce que l'autre lui propose et elle ne manifeste aucune demande. Son absence de réactivité à la présence des autres et à toute proposition la rend comme inconsistante tel un être transparent et inexistant. J'ai parfois l'impression qu'elle est en état de liquéfaction, comme une flaque d'eau. Elle s'allonge souvent par terre pour colmater le vide sous la porte. Un corps sans âme ou une âme sans corps.

Son absence de réaction à l'autre nous rend comme inutiles, transparents ou inexistants. J'ai le sentiment de temps en temps d'être moi-même un fantôme sans forme perceptible, hors temps et hors corps. Mais je reste confiante dans l'espoir que le lien pourrait être bénéfique à Léa avec de la patience malgré son absence de manifestation. Je raconte ce que j'imagine 
qu'elle peut sentir, éprouver ou penser à chaque changement, chaque mouvement, autant dans ses éprouvés internes que dans ceux venant de son environnement, afin qu'elle retrouve un sentiment d'exister dans le lien à l'autre.

\section{DÉPLACEMENTS ET MOUVEMENTS}

Ses déplacements ne s'accordent pas du tout aux mouvements ou aux déplacements des autres. Son corps sans tonus est comme bloqué, sans aucune souplesse. Elle avance tête en avant comme un automate qu'il faut soulever pour sa mise en route et qui ne s'arrête devant aucun obstacle, mue par un mouvement lent quasi automatique et à vitesse constante dès lors qu'elle a commencé sa trajectoire. Le bas de son corps n'est pas habité. Elle n'ajuste pas sa marche aux aspérités du sol. Son regard est absent et flottant, tel un zombi ou un fantôme. Elle se cogne sur les obstacles rencontrés, comme si son corps et les objets de son environnement n'avaient aucune densité et sans réflexe d'évitement. Je la réoriente au cours de ses déplacements comme je le ferais avec une personne aveugle.

La différence entre le mode de déplacement des humains et celui des objets non humains est qu'un objet inerte ne peut se déplacer que si un autre corps le heurte et déclenche son déplacement. À moins que cet objet inerte subisse la loi de la gravitation pour tomber s'il est en déséquilibre ou qu'il soit un objet mécanique remonté pour qu'il s'anime. Un humain peut se déplacer spontanément sans avoir besoin de recevoir une impulsion sur son corps pour déclencher son mouvement. Une émotion peut le mobiliser et déclencher un mouvement spontané sur son visage ou dans son corps.

Léa semble déshabiter son corps et réagit comme un objet inerte qui se déplacerait par une impulsion que nous lui aurions donnée pour avancer et qui ne s'arrêterait que s'il heurte un autre objet sur son passage, sans qu'il puisse ajuster sa trajectoire avec sa vision ou au contact d'un autre.

Les personnes autistes ont une très mauvaise perception du mouvement et des déplacements, ce qui leur procure beaucoup d'angoisses dans l'espace. Laurent Mottron (2004) a montré qu'il faut percevoir en même temps plusieurs points en mouvement pour appréhender le mouvement d'un corps dans sa globalité. Bon nombre de personnes autistes s'agrippent à un détail sans pouvoir passer avec souplesse à une vision globale. Elles ne peuvent pas suivre plusieurs points en mouvement à la fois. Ainsi, la perception des mouvements imprévisibles et trop rapides leur est très difficile et devient source d'angoisse.

Les angoisses spatiales peuvent se déclencher au cours de leurs déplacements dans des espaces trop ouverts, sans bords qui pourraient les contenir. 
Elles tentent de maîtriser à leur façon cette énigme qu'est le mouvement dans l'espace, qui les fascine et leur fait peur, en étant attirées par tous les objets en mouvement comme les portes automatiques ou les ascenseurs. D'autres font les acrobates ou marchent en arrière à toute vitesse sur un sol en pente (Tammet, 2006) pour tenter d'étudier les lois de la gravitation et des mouvements de leur corps dans l'espace.

\section{RALENTISSEMENT ET VITESSE}

Bruno Gepner (2001) a étudié les troubles de la perception visuelle des mouvements chez les personnes autistes. Il conseille judicieusement de ralentir les mouvements de nos déplacements et de notre parole à leur contact.

Léa ralentit spontanément ses mouvements et ses déplacements en adoptant une vitesse lente et constante. Étirer le temps par le ralentissement l'aide à diminuer ses angoisses spatiales et à réguler les intensités émotionnelles qui l'animent.

La perception de la profondeur de champ, cette troisième dimension de l'espace, est très difficile tant que la relation n'est pas installée par l'échange des regards. Le corps n'est pas vécu en volume tant que la mise en place de la contenance dans la relation n'est pas possible et que le bas du corps est peu construit. Les vertiges dans l'espace sont ainsi fréquents. Les angoisses d'effondrement sont diminuées par l'immobilité ou par le ralentissement.

L'identification à Léa et la rencontre avec elle ne sont possibles que si je me mets à son rythme extrêmement ralenti et délicat et si mes mouvements sont fluides.

Je lui prête ma rêverie à propos de ses mouvements, ses déplacements, ses balancements ou son immobilité et des sensations qui la traversent. Je lui parle des sensations qui lui parviennent sans qu'elle puisse les exprimer ou y réagir, de son environnement peuplé d'objets, de limites, de murs, de porte, de table ou de chaise, de lumières, de contrastes ou de mouvements de pensées ou d'émotions à chaque changement. Je raconte la solidité des points de butée au hasard de ses déplacements pour donner consistance et surface aux objets qu'elle rencontre.

\section{RÉACTIONS À LA DOULEUR}

Peu à peu elle s'agrippe à mon bras pendant les trajets. Léa ne réagit pas à mes commentaires dans un premier temps, mais quand je suis assise, elle vient me présenter son dos. Je deviens comme un bon dossier de chaise. Je l'installe alors sur mes genoux. Ce contact de surface d'arrière-plan semble 
lui redonner consistance. La confiance s'installe ainsi qu'une familiarité par ma présence et par ma voix probablement. Elle commence à tapoter les surfaces du bout de ses doigts comme pour éprouver des rencontres avec un environnement qui devient plus consistant, tel un aveugle qui explore l'espace avec sa canne en tapotant les surfaces autour de lui pour se frayer un passage sécurisé.

Mes commentaires sur ses éprouvés, qu'ils soient potentiellement agréables ou douloureux, ne provoquent aucune réaction chez Léa pendant quelques années.

Sylvie Torjmann a mis en évidence que les réactions à la douleur chez certaines personnes autistes ne passent ni par une manifestation secondaire ni par un réflexe de retrait ou de fuite, mais par des réactions plus profondes neurovégétatives comme la tachycardie, la transpiration, les productions $\mathrm{d}$ 'hormones de stress qui sont supérieures à celles que nous aurions dans les mêmes circonstances.

Je commente et exprime la douleur en en théâtralisant l'expression quand Léa se cogne, par identification à son vécu profond comme le ferait une mère préoccupée qui commenterait à son bébé ce qu'il vit dans son corps avant qu'il acquière la parole.

Par la suite elle est capable de tapoter les zones douloureuses de son corps, comme je le lui ai souvent proposé de le faire à partir d'un geste qu'elle avait déjà expérimenté sur d'autres surfaces. Elle a pu ainsi manifester directement sa douleur en s'accompagnant de petits geignements et de quelques grimaces.

\section{STÉRÉOTYPIES VERS LA LUMIÈRE}

Quand elle est debout, elle reste figée sur place dans le bas de son corps et se tourne dans la direction du soleil, de la lumière ou d'un contraste lumineux. Elle adopte alors une stéréotypie qui consiste à aligner les doigts de sa main gauche devant son visage en les animant de micromouvements constants. Elle fixe ses doigts d'un regard légèrement sur le côté dans la direction du contraste lumineux, tout en tapotant ses dents avec les ongles de sa main droite. S'agit-il de trouver ainsi un bord dans l'espace afin de diminuer ses angoisses spatiales ? Elle évite le regard direct. Son regard est autrement dans le vide. Ainsi, elle semble maintenir son sentiment d'existence par les vibrations qu'elle se procure de façon autistique en se tapotant les dents et qui résonnent dans toute sa tête.

De loin, avec une confiance qui s'installe dans nos rencontres, elle commence à déplacer puis remplacer son intérêt pour les lumières ou les contrastes en dirigeant son visage dans la direction du mien tout en conti- 
nuant à interposer ses doigts ou des objets en micromouvements entre nos regards. Je sens parfois qu'elle réussit à focaliser son regard sur mon visage, d'abord en interposant entre nous ses stéréotypies, puis directement en abandonnant ses stéréotypies. Je m'aperçois rapidement que ce n'est pas encore mon visage qui l'intéresse mais mon collier de perles blanches brillantes, sans doute associé à la provenance de ma voix et de mon attention pour elle.

C'est l'attirance vers les contrastes lumineux des perles blanches de mon collier qui a permis à Léa de se diriger spontanément vers moi. Je mettrai par la suite ce collier quand je la recevrai et le lui confierai volontiers au moment où elle s'y agrippera. Elle alterne de façon compulsive deux mouvements avec le collier. Elle le tend à l'horizontale tout en observant chaque perle. Elle le ramasse ensuite sur lui-même du bout des doigts avec ses deux mains dans un mouvement symétrique en entrechoquant l'amas de perles. Puis elle recommence à le tendre à l'horizontale et l'observe attentivement pour ensuite l'amasser de nouveau.

Malgré la répétitivité des gestes, ces manœuvres sont sources pour moi de rêveries variées dont je lui fais part en fonction des événements traversés et de nos rencontres. Mes commentaires sur l'émerveillement et la fascination que j'ai l'impression qu'elle éprouve face à ces perles rondes, brillantes, magnifiques, bien alignées, accrochées ensemble tout en étant différenciées, semblent lui convenir. Je sens Léa plus concernée par elle-même puis par le lien qui s'établit entre nous. En effet, elle dirige peu à peu de plus en plus souvent le collier non plus vers une source lumineuse prise dans l'environnement non humain mais dans ma direction d'où provient ma voix. Par la suite, elle m'observera particulièrement quand je ne la regarderai pas et elle abandonnera ses stéréotypies avec le collier pour être plus présente.

Je rencontre régulièrement sa mère avec elle. Madame est une mère aimante, dévouée et très investie auprès de ses deux enfants. Léa ne réagit à rien malgré l'attention de sa mère. Celle-ci parle pourtant de sa fille avec bienveillance et ses yeux sont pétillants. Nous évoquons la dépression profonde dans laquelle elle était aux alentours de la naissance de Léa et dont elle est sortie. Léa traverse la pièce pour se diriger vers mon collier de perles qu'elle agrippe jusqu'à ce que je le lui prête. Madame me confie que sa fille ne l'a jamais encore regardée depuis sa naissance, c'est-à-dire depuis douze ans. Elle en est attristée. J'en suis bouleversée tandis que je commente à Léa que les perles sont aussi jolies que le regard pétillant de sa maman, et j'ajoute que sans doute elle n'a jamais su comment se diriger vers les yeux de sa maman. Léa abandonne alors mon collier, déambule doucement vers sa maman, tente de se mettre en position fotale collée au bras gauche de sa mère, lève la tête sur le côté comme un nourrisson au sein et se plonge pour 
la première fois dans le regard maternel pendant un long moment. Sa mère est émue aux larmes. Moi aussi. Par la suite, des échanges de regard s'installent peu à peu, dans de brefs instants et de loin ou suite à mes commentaires. Elle se déplace plus facilement dans l'espace.

\section{AJUSTEMENTS DANS L'ESPACE, MOUVEMENTS ET REGARD PÉRIPHÉRIQUE}

Tant qu'une personne a des angoisses spatiales, elle préfère utiliser le regard périphérique plutôt que le regard en direct. Le regard périphérique, d'après les travaux d'André Bullinger (2005), a pour fonction de permettre un ajustement du corps dans l'espace, particulièrement quand le regard en face n'est pas encore investi dans la dimension de la profondeur de champ. Les contrastes en mouvement perçus en regard périphérique stabilisent le corps dans l'espace et font diminuer les vertiges et les craintes d'effondrement, comme si l'espace environnemental alors bordé par ces flux visuels devenait contenant.

Quand Léa regarde de biais l'alignement de ses doigts à la verticale ou des perles à l'horizontale, elle provoque des contrastes lumineux géométriques en mouvement rassurants.

Elle agite également ses mains sur les côtés de son visage, comme pour tenter de s'équilibrer dans l'espace, alors que le bas du corps reste figé et immobile, et que ses poignets encore mal reliés aux autres parties de son image du corps sont la plupart du temps sans aucun tonus.

Probablement Léa se sent-elle en sécurité en se balançant d'avant en arrière. Car, outre le confort du rythme primordial cardiaque maternel obtenu, ses mouvements lui procurent également des flux visuels qui la rassurent dans l'espace.

De même, ses balancements de droite à gauche la stabilisent. Et ces allers et retours forgent une charnière verticale entre l'hémicorps droit et l'hémicorps gauche, comme l'a décrit Geneviève Haag (1983). Par la suite elle arrêtera de se balancer.

Léa apprécie les commentaires sur ses manifestations corporelles, sensorielles ou émotionnelles, même si elle n'a par la suite jamais développé de langage verbal articulé. Elle y réagit en différé dans les premières années de suivi et plus en phase par la suite. Son regard devient plus souvent vivant, présent, adressé et moins flottant. Elle commence à suivre des conversations, ce qu'elle manifeste par quelques poursuites oculaires vers ses interlocuteurs et par des esquisses de sourires.

Sa mère est contente et même amusée de la voir capable de râler quand quelque chose ne lui convient pas ou d'esquisser de larges sourires quand elle est satisfaite. Léa apprécie davantage la compagnie, les distractions et les 
allées et venues des uns et des autres. Elle n'est plus transparente comme un fantôme, mais elle a l'allure d'une adolescente. Elle compte désormais pour les autres et les autres semblent compter pour elle.

\section{ACCÉLÉRATION ET ANIMATION DU VISAGE}

Peu à peu, quand je viens la chercher pour sa séance, elle se lève spontanément avec un sourire de contentement. Son corps est plus tonique avec moins d'autoagrippements musculaires. Elle essaie de marcher avec plus d'entrain même si ses jambes sont encore raides. Ses petits pas semblent s'accélérer à la mesure de notre plaisir à nous retrouver jusqu'à ce qu'elle s'exerce à faire de grands pas plus efficaces de façon rythmique. Elle me reconnait et se sent en sécurité avec le son de ma voix qui lui est désormais familier.

Par la suite, je la surprends, en entrant dans le bureau, à se précipiter à petits pas pour s'installer dans mon fauteuil. Elle dirige son regard vers la chaise où elle a l'habitude de s'asseoir contre le mur. Je lui demande si elle essaie de voir comment je la vois. Si son corps est plus mobile, je constate que son visage immobile semble encore fantomatique. Je lui propose de rester dans mon fauteuil sans la déloger. J'accroche une grande feuille sur le mur derrière sa chaise habituelle et je fais son portrait en grand format tel que je la vois d'habitude en prenant le soin de nommer chaque partie de son visage. Pendant que je suis censée ne pas la regarder, je la surprends discrètement en train de regarder dans ma direction. Je fais dans les séances suivantes une série de portraits en continuant de nommer chaque partie du visage au fur et à mesure.

Elle caresse souvent le haut de sa joue à la suite de mes commentaires, ce qui déclenche un réinvestissement de sa zone orale et un réveil des réflexes d'ouverture de sa bouche. Son visage commence à s'animer davantage en commençant par des tics et des grimaces. Ses expressions faciales se dégèlent peu à peu et elle devient plus expressive.

Je ne manque pas de nommer les sensations éprouvées sur les lèvres puis sur le menton provoquées par sa salive qui coule malgré elle en dehors de sa bouche. Je passe de nombreuses séances à nettoyer son pull de ses traînées de salive pour qu'elle dégoûte moins son entourage pendant les quelques heures qui suivent les séances.

Sa bouche se réanime, elle devient plus tonique et j'ai le sentiment qu'elle commence à exister. Je vois alors Léa essuyer sa bouche d'un revers de manche, après des commentaires que je fais sur sa bouche. Elle tente d'avaler sa salive et elle arrêtera de baver par la suite.

Elle commence à émettre quelques petits sons qui accompagnent progressivement l'expression naissante de ses émotions sur son visage. 


\section{EN CONCLUSION : LE LIEN AVEC LES ÊTRES FANTOMATIQUES}

Il est toujours troublant de voir combien le maintien du lien adressé pendant des années à une personne en retrait qui ne manifeste rien construit un sentiment d'exister en profondeur. La patience, l'attention et les commentaires identifiants du thérapeute, bienveillant en continu, sur des sensations et des émotions éprouvées par une personne autiste qui n'exprime aucune réponse dans un premier temps ont pour effet d'animer des parties encore immobiles et peu vivantes qui avaient renoncé au lien. L'accordage à son rythme extrêmement lent est une condition d'accès à une rencontre possible.

Les êtres fantomatiques seraient-ils fortement présents quand la vie n'est plus traversée par la possibilité de rencontre et d'accordage émotionnels avec un autre ? La nostalgie du lien avec des personnes qui ne sont plus de ce monde ou avec des expériences archaïques in utero tenterait alors de garder un contact subtil mais impalpable, inconsistant, au point de nous mettre dans des états de transparence hors espace, hors temps, hors corps, où la vitesse des mouvements du vivant ne s'accorde plus provisoirement aux mouvements des êtres sociaux plus incarnés.

Nos figurations fantasmatiques des fantômes seraient des états intermédiaires entre les parties mortes d'un corps déshabité et le mouvement de la vie avec des allures humaines qui ne demandent qu'à exister puis à se manifester dans la relation à l'autre.

\section{BIBLIOGRAPHIE}

BULlinger, A. 2005. Le développement sensori-moteur de l'enfant et ses avatars. Un parcours de recherche, Toulouse, érès.

GEPNER, B. 2001. " "Malvoyance" du mouvement dans l'autisme infantile ? Une nouvelle approche neuropsychopathologique développementale », La psychiatrie de l'enfant, 1, vol. 44, Paris, PUF, 77-126.

HAAG, G. 1985. « La mère et le bébé dans les deux moitiés du corps », Neuropsychiatrie de l'enfance, 33 (2-3) [deuxième congrès mondial de psychiatrie du nourrisson, Cannes, 1983], 107-114.

Le Poulichet, S. 2010. Les chimères du corps, Paris, Aubier.

LheUREuX-DAVIDSE, C. 2003. L'autisme infantile ou le bruit de la rencontre, Paris, L'Harmattan.

LHEUREuX-DAVIDSE, C. 2004. " La reconstruction de l'image du corps de Léo, enfant trisomique avec des retraits autistiques », Cliniques méditerranéennes, 69, 289-308.

LHEUREUX-DAVIDSE, C. 2007. « Jouer avec les mouvements, les vibrations et les rythmes dans l'émergence de la voix », Champ psychosomatique, 48, 185-203. 
LHEUREuX-DAVIDSE, C. 2009. «Vécus corporels chez des personnes autistes. La place du corps et de la sensorialité dans les installations en clivage », dans Le corps vécu chez les personnes âgées et les personnes handicapées, Paris, Dunod, coll. "Action sociale ».

MotTron, L. 2004. L'autisme: une autre intelligence. Diagnostic, cognition et support des personnes autistes sans déficience intellectuelle, Sprimont, Pierre Mardaga éditeur.

RACAMIER, P.-C. 1992. Le génie des origines. Psychanalyse et psychoses, Paris, Payot, coll. «Bibliothèque scientifique ».

TAMMET, D. 2006. Je suis né un jour bleu, Paris, Éditions des arènes.

TORJMAN, S. 2004. «Étude longitudinale des relations entre l'apparente insensibilité à la douleur et les troubles de la communication non verbale et de la symbolisation dans l'autisme », Contraste, Enfance et handicap, 21.

TustiN, F. 1986. Autistic Barriers in Neurotic Patients, Londres, Karnac Books, trad. Paul Chemla.

TUSTIN, F. 1989. Le trou noir de la psyché, Barrières autistiques chez les névrosés, Paris, Le Seuil.

WinNiCOTT, D. 1975. « La crainte de l'effondrement » (trad. J. Kalmanovitch et M. Gribinski), Nouvelle revue de psychanalyse, 11, 35-44.

Résumé

Les représentations que nous nous faisons des fantômes nous éclairent sur les états flottants des personnes peu habitées dans leur corps et peu investies dans les relations en direct. Chez des personnes absorbées par un deuil ou dans des cas de dépression, ou chez des personnes en retrait autistique, la perception de mouvements trop rapides ou imprévisibles et des déplacements dans l'espace provoquent parfois des angoisses spatiales conséquentes qui ne les rendent plus disponibles à la relation.

Afin qu'une identification et qu'une rencontre soient possibles, l'accordage du thérapeute à leur rythme souvent très lent et délicat ainsi que les commentaires sur les vécus internes peuvent réguler des vécus trop intenses ou clivés aux niveaux tant sensoriel et émotionnel que relationnel. Chez des personnes comme transparentes qui ne peuvent encore se manifester, les réactions se font d'abord en différé avant qu'elles soient en synchronie.

Mots-clés

Vitesse, déplacement, transparence, fantômes, corps, autisme, espace.

RAPIDITY, MOVEMENT AND TRANSPARENCY OF PHANTOM BEINGS AND AUTISTIC PERSONS

\section{Summary}

The representations we make of ghosts can illuminate us on the floating state of persons who are not very connected with their body and who don't invest in direct relationships. For persons absorbed in mourning, or cases of depression or persons with autistic characteristics, the perception of movements that are too rapid or unpre- 
dictable and changing of place can provoke spacial fears with the consequence that they are no longer available for the relation.

An identification and relationship become possible if the therapist puts him or herself in the same very slow and delicate rhythm. Comments on the interior experiences help to regulate when it is too intense or splitting, which can be at a sensory, emotional or relationship level. For persons that are as if they are transparents and can not manifest themselves, the reactions are at first at a later moment, before they become synchronized.

Keywords

Rapidity, movement, transparency, ghosts, body, autism, space. 